\title{
Epigenetic modifications acetylation and deacetylation play important roles in juvenile hormone action
}

\author{
Amit Roy ${ }^{1,2}$ and Subba Reddy Palli ${ }^{*}$
}

\begin{abstract}
Background: Epigenetic modifications including DNA methylation and post-translational modifications of histones are known to regulate gene expression. Antagonistic activities of histone acetyltransferases (HATs) and histone deacetylases (HDACs) mediate transcriptional reprogramming during insect development as shown in Drosophila melanogaster and other insects. Juvenile hormones $(\mathrm{JH})$ play vital roles in the regulation of growth, development, metamorphosis, reproduction and other physiological processes. However, our current understanding of epigenetic regulation of $\mathrm{JH}$ action is still limited. Hence, we studied the role of CREB binding protein (CBP, contains HAT domain) and Trichostatin A (TSA, HDAC inhibitor) on JH action.

Results: Exposure of Tribolium castaneum cells (TcA cells) to JH or TSA caused an increase in expression of Kr-h1 (a known JH-response gene) and 31 or 698 other genes respectively. Knockdown of the gene coding for CBP caused a decrease in the expression of 456 genes including $\mathrm{Kr}-\mathrm{h} 1$. Interestingly, the expression of several genes coding for transcription factors, nuclear receptors, P450 and fatty acid synthase family members that are known to mediate $\mathrm{JH}$ action were affected by CBP knockdown or TSA treatment.
\end{abstract}

Conclusions: These data suggest that acetylation and deacetylation mediated by HATs and HDACs play an important role in $\mathrm{JH}$ action.

Keywords: HAT, HDAC, Kr-h1, FOXO Tribolium and TCA cells

\section{Introduction}

Immature juvenile forms of animals transform to become reproductive adults during their life cycle. In insects, two types of such transformation are generally observed; incomplete metamorphosis where nymphs turn into adults in hemimetabolous insects (e.g. true bugs, locusts \& cockroaches) and complete metamorphosis which comprises dramatic morphological changes that result in the transformation from larva to the intermediate form, pupa and then to reproductive adults as observed in holometabolous insects (e.g. beetles and butterflies) [1]. Despite the differences in the strategies used by the insects, the genetic switch between different forms is tightly regulated by changes in the levels of two major hormones, ecdysteroids (20-hydroxyecdysone, 20E is the most active form) and

\footnotetext{
* Correspondence: rpalli@uky.edu

'Department of Entomology, College of Agriculture, Food and Environment, University of Kentucky, Lexington, KY 40546, USA

Full list of author information is available at the end of the article
}

juvenile hormones (JH) [2, 3]. 20-hydroxyecdysone works through its receptor complex, ecdysone receptor (EcR) and ultraspiracle (USP) that binds to ecdysone response elements (EcRE) present in the promoter regions of ecdysone-response genes including ecdysone-induced transcription factors ("early genes") and others [4, 5]. Periodic pulses of 20E ensure developmental transitions during molting and metamorphosis. The presence of $\mathrm{JH}$ prevents metamorphosis, and the absence of $\mathrm{JH}$ promotes metamorphosis. Juvenile hormones function through its receptor, Methoprene-tolerant (Met) and steroid receptor coactivator (SRC, also known as FISC or Taiman). The JH/MET/SRC complex binds to JH response elements (JHRE) present in the promoter regions of JH-response genes including Krüppel homolog $1(K r-h 1)$ and plays key roles in "status quo" action of JH $[6,7]$. In Bombyx mori, $\mathrm{Kr}$-h1 functions as a direct transcriptional repressor of E93, the adult specifier gene, through binding to response elements present in the promoter region of this gene [8].

(c) The Author(s). 2018 Open Access This article is distributed under the terms of the Creative Commons Attribution 4.0 International License (http://creativecommons.org/licenses/by/4.0/), which permits unrestricted use, distribution, and 
In addition to signaling through nuclear receptors and transcription factors, JH may also function through membrane receptors and second messengers $[9,10]$. Our previous work in T. castaneum has documented the dopamine D2-like receptor [G-protein-coupled-receptor (GPCR) family member] playing critical roles in regulating JH-mediated Vg uptake [11]. Cai et al., [12] reported the involvement of GPCRs during the 20E signaling suggesting that membrane receptors may be involved in this hormone signaling. Furthermore, it has been proposed that $\mathrm{JH}$ could regulate $\mathrm{Ca}^{2+}$ homeostasis within the cell during metamorphosis by interacting with membrane receptors or ion channels. Previously, farnesol (a precursor of $\mathrm{JH}$ ) was shown to block $\mathrm{Ca}^{2+}$ channels [13]. It has been suggested that the JH maintains the "status quo effect" by reducing the cellular $\mathrm{Ca}^{2+}$ levels and when $\mathrm{JH}$ titers decline, such regulation is released, and the cellular $\mathrm{Ca}^{2+}$ levels are elevated to induce apoptosis, a process that occurs during metamorphosis [13].

In addition to metamorphosis, $\mathrm{JH}$ also regulates other crucial aspects of insect life including reproduction, diapause and caste differentiation [14]. In D. melanogaster females, JH controls yolk protein synthesis in the fat body and ovaries [15]. In males, JH regulates courtship behavior and promotes synthesis of accessory gland proteins [16-18]. The regulatory mechanisms underlying orchestrated synthesis of $\mathrm{JH}$ and expression of its receptors and target genes including transcription factors, co-activators for regulation of various physiological processes such as molting and metamorphosis are not yet precise.

Recent studies documented the role of CBP (or Nejire), a protein with acetyltransferase activity (HAT), as a transcriptional co-regulator in various developmental processes in D. melanogaster $[19,20]$. In addition, the circadian rhythms in D. melanogaster also rely on the presence of Nejire/CBP [21]. Intriguingly, with more than 400 documented interacting partners mostly belonging to transcription factor and growth regulator families, $\mathrm{CBP}$ acts as a hub for transcriptional network involved in regulation of hormone signaling pathways and other fundamental physiological processes in insect life [19, 22, 23]. Recently, the involvement of CBP in the metamorphosis of Blatella germanica was reported [24]. The HAT activity of CBP increases acetylation of histones resulting in neutralization of lysine residues, which in turn increases accessibility of the promoters to cellular transcriptional machinery resulting in higher gene expression.

Histone deacetylases (HDACs) remove acetyl groups, increasing DNA and histone interactions resulting in repression of target gene expression through diminishing the accessibility of the promoters to transcription factors. Hence, stringent regulation of DNA packaging by HATs and HDACs can control gene expression. In insects,
HDAC inhibitor activity might facilitate caste specification behavior in honey bees [25]. Epigenetic control of neural plasticity in honey bee has been documented recently [26]. Furthermore, the size and their nutrition-dependent hyper-variability of mandibles in the broad-horned flour beetle, Gnatocerus cornutus, are regulated by HDACs. HDAC1 RNAi in the beetle larvae caused shortening of mandibles in the adults, whereas HDAC3 RNAi resulted in hypertrophy [27]. In the case of ants, the caste-specific foraging and scouting behaviors are also regulated epigenetically by the equilibrium between CBP-mediated acetylation and HDAC-mediated deacetylation of histones in the brain [28].

However, only limited information on the contribution of HATs or HDACs to $\mathrm{JH}$ action is available. In the current study, we elucidated the effect of CBP and an HDAC inhibitor TSA on gene expression using TcA cells as a model system because it has already served as a robust research platform for molecular analysis of $\mathrm{JH}$ signaling pathway $[29,30]$. The expression of CBP was knocked down by exposing TcA cells to $d s C B P$. Exposure of $C B P$ knockdown cells to DMSO (control), JH or TSA followed by sequencing of RNA isolated from the treated cells revealed the influence of acetylation status of histones on the expression of genes involved in $\mathrm{JH}$ action.

\section{Materials and methods}

\section{Cell culture}

The Tribolium castaneum cells (BCIRL-TcA-CLG1 are gift from Dr. Cynthia Goodman from Biological control of insects research laboratory, USDA-ARS, $1503 \mathrm{~S}$. Providence, Columbia, MO, USA) were grown at $28^{\circ} \mathrm{C}$ in Ex-cell 420 (Sigma) supplemented with 10\% Fetal Bovine Serum (Life Technologies) following the protocol described recently [31].

\section{Knockdown of the CBP gene}

Double-stranded RNA targeting $C B P$ gene $(d s C B P)$ was synthesized using the cDNA template and MEGAscript RNAi kit $\left(\right.$ Ambion $\left.^{\mathrm{Tx}}\right)$. For knockdown experiments, TcA cells were seeded in 12-well plates. After overnight culture in the medium containing 10\% FBS, the cells were treated with either $1 \mu \mathrm{g}$ of $d s C B P$ or dsmalE (as control dsRNA targeting a fragment of $E$. coli maltase gene) for $72 \mathrm{~h}$. At the end of the incubation with dsRNA, the cells were treated with JH III or TSA dissolved in DMSO. DMSO was added to the control cells. After $6 \mathrm{~h}$ of hormone treatment, the TcA cells were washed with $1 \mathrm{X}$ PBS, harvested and processed for isolation of RNA.

Total RNA was isolated using the TRI reagent (Molecular Research Center Inc., Cincinnati, OH). The DNA was eliminated from the total RNA using DNase I (Ambion Inc., Austin, TX). RNA quantity was measured using a NanoDrop 2000 spectrophotometer (Thermo 
Fisher Scientific), and quality was determined by running on 1\% agarose gel. Two micrograms of total RNA from each sample was used for cDNA synthesis. cDNA was used to amplify a fragment of the target gene, and the PCR product was used for dsRNA synthesis. Primers used for dsRNA synthesis and reverse-transcriptase quantitative real-time PCR (RT-qPCR) are listed in Additional file 1: Table S1 or reported in our previous publication [32]. The MEGAscript RNAi Kit (Ambion Inc., Austin, TX) was employed for dsRNA synthesis. After total RNA extraction, cDNA synthesis and RT-qPCR were performed following the protocol described previously [33].

\section{RNA-seq study design, library preparation, and sequencing}

Total RNA was extracted from dsmalE (M) treated cells exposed to DMSO (dsmalE+ DMSO or MD), JH III (dsmalE+ JHIII or MJ) or TSA (dsmalE+ TSA or MT) and dsCBP $(C)$ treated cells exposed to DMSO (dsCBP+ DMSO or CD), JH III $(d s C B P+\mathrm{JHIII}$ or CJ) or TSA $(d s C B P+\mathrm{TSA}$ or $\mathrm{CT})$. Three biological replicates were used for each treatment. The design of RNA seq experiment is presented in Table 1. The protocol used for RNA seq library preparation include oligo-dT based total RNA separation, RNA fragmentation, two-step cDNA preparation and library barcoding, as described previously [33, 34]. Briefly, mRNA was purified and enriched from $3 \mu \mathrm{g}$ of high-quality RNA from each biological replicate, fragmented and used for cDNA preparation. First strand cDNA synthesis was performed using SMARTSCribe reverse transcriptase enzyme (Clontech Laboratories, Inc) and reverse transcriptase primers (Integrated DNA technologies) with a sequencing adaptor followed by a random hexamer for Illumina sequencing and unique barcode for multiplexing. Second strand cDNA was synthesized using SMART 7.5 primer (Clontech Laboratories, Inc). The cDNA fragments were size selected ( $300 \mathrm{bp})$ using HighPrep TM PCR beads (MAGBIO) and finally amplified using PCR. Libraries

Table 1 RNA-seq study design

\begin{tabular}{lll}
\hline No & Comparisons & Abbreviation \\
\hline 1 & $\begin{array}{l}\text { DMSO and JH treated cells } \\
\text { exposed to dsmalE }\end{array}$ & MD vs MJ \\
2 & $\begin{array}{l}\text { DMSO treated cells exposed } \\
\text { to dsmalE or dsCBP }\end{array}$ & MD vs CD \\
3 & $\begin{array}{l}\text { DMSO or JH treated cells } \\
\text { exposed to dsmalE or dsCBP }\end{array}$ & MD vs CJ \\
4 & $\begin{array}{l}\text { DMSO or TSA treated cells } \\
\text { exposed to dsmalE or dsCBP }\end{array}$ & MD vs CT \\
5 & $\begin{array}{l}\text { DMSO or TSA treated cell } \\
\text { exposed to dsmalE }\end{array}$ & MD vs MT \\
\hline
\end{tabular}

The table shows a list of comparisons made after RNA sequencing. All the treatments are compared with the control (MD) are quantified by Nanodrop and verified by running them on $1.2 \%$ agarose gels. Finally, the libraries were multiplexed and sent for sequencing (single-end) using the HiSeq4000 sequencer at the Genomics Technologies Center of Duke University, NC, USA. Raw sequencing data statistics are presented in Table 2 .

\section{RNA-seq data analysis}

Raw reads after quality control (demultiplexing, trimming and adaptor removal) were mapped to the reference genome (GA-2 strain of T. castaneum) [35]. The parameters used for the mapping are mismatch $\operatorname{cost}=2$, insertion $\operatorname{cost}=3$, deletion cost $=3$, length fraction $=0.8$, similarity fraction $=0.8$, unique exon read mapping. The read counts were $\log _{10}$ transformed followed by normalization using the scaling method where the sets of the transformed expression values for the samples were multiplied by a constant so that the sets of normalized values for the samples had the same 'target' value. The RNA-seq tool from CLC workbench (Version 9.5.9) was used to quantify gene expression employing standard pre-optimized settings and parameters such as mapping to exon regions only [36]. Further, biases in the sequence datasets and different transcript sizes were corrected using the RPKM algorithm to obtain correct estimates for relative expression levels. Finally, EDGE (Empirical analysis of differential gene expression) analysis was performed through "Advanced RNA-Seq plug-in" in CLC workbench using the recommended parameters such as estimated count filter cut off 5, estimate tag-wise dispersion [33]. For differential gene expression, we set a $p$-value cut-off of $<0.01$ and fold change cut-off of \pm 2 -fold as a threshold value for being significant. Differentially expressed genes were functionally annotated using "cloud blast" feature within the "Blasto2GO plug In" in CLC Genomic Workbench (Version 9.5.9). Nucleotide blast was performed against arthropod database with an E-value cut off 1.0E-3. Both, annex and GO slim were used to improve the GO term identification further by crossing the three GO categories (biological process, molecular function, and cellular component) to search for name similarities, GO term and enzyme relationships within KEGG (Kyoto Encyclopedia of Genes and Genomes) pathways.

\section{RT-qPCR validation}

RT-qPCR was conducted to validate the RNA seq results with a subset of 15 genes induced after $\mathrm{JH}$ treatment. Primers were designed using IDT's primer design software (www.idtdna.com) with parameters including length $18-25 \mathrm{nt}$, melting temperature $55-65^{\circ} \mathrm{C}, \mathrm{GC}$ content $50-60 \%$ and product size $100-150 \mathrm{bp}$ (Additional file 1: Table S1). Primers were tested by PCR followed by gel electrophoresis for correct product amplification. cDNA for RT-qPCR were synthesized using RNA from a 
Table 2 RNA-seq statistics

\begin{tabular}{|c|c|c|c|c|c|c|}
\hline \multirow[b]{2}{*}{ Samples } & \multicolumn{3}{|l|}{ dsmalE } & \multicolumn{3}{|l|}{$\mathrm{dsCBP}$} \\
\hline & MD1-MD3 & MJ1-MJ3 & MT1-MT3 & CD1-CD3 & CJ1-CJ3 & $\mathrm{CT1-CT3}$ \\
\hline Total no. of reads & $90,280,120$ & $64,052,276$ & $86,015,875$ & $98,406,728$ & $100,327,204$ & $94,045,683$ \\
\hline No. of high-quality reads & $80,437,710$ & $57,466,708$ & $54,928,048$ & $86,047,960$ & $79,067,179$ & $79,188,207$ \\
\hline Mapped reads to Exon ${ }^{a}$ & $75,27,476$ & $53,578,082$ & $48,830,575$ & $81,330,901$ & $73,271,933$ & $71,579,430$ \\
\hline Percent reads mapped & 83.59 & 93.29 & 87.89 & 93.36 & 92.97 & 90.37 \\
\hline Uniquely mapped reads ${ }^{\mathrm{b}}$ & $70,309,618$ & $49,895,642$ & $45,547,725$ & $75,753,713$ & $68,994,581$ & $67,063,490$ \\
\hline
\end{tabular}

${ }^{a}$ Exon regions of Tribolium castaneum genome

${ }^{\mathrm{b}}$ Reads considered for downstream gene expression analysis

The table is showing a summary of read statistics after Illumina Hi-seq 4000 sequencing. Total read counts for three biological replicates from each treatment varies within $64,000,000$ to $101,000,000$. Number of high quality reads indicates the number of reads left after read trimming and filtering (Quality control step). Percentage of high quality reads unambiguously mapped to reference Tribolium genome represented in the second last row. Uniquely mapped reads were considered for differential gene expression analysis. Further details of RNA-seq statistics per sample are given in Additional file 1: Table S2

new set of dsmalE, and dsCBP treated samples. cDNA was prepared from $3 \mu \mathrm{g}$ RNA per sample using M-MLV reverse transcriptase kit (Invitrogen TM) following the manufacturer protocol. Resulting cDNA samples were diluted (4 times), and RT-qPCR was performed using SYBR Green Supermix (Bio-rad) following existing lab protocol [33]. Melt curves were generated to ensure single product amplification. The target genes expression levels were calculated using the 2- $\Delta \mathrm{Ct}$ method with RP49 (a ribosomal protein) as a reference gene, which had already been validated as a housekeeping gene in Tribolium castaneum [37-39].

\section{Results and discussion}

Ecdysteroids and $\mathrm{JH}$ regulate insect metamorphosis by performing antagonistic roles [40]. 20E facilitates metamorphosis whereas the presence of $\mathrm{JH}$ prevents it until the appropriate time. The role of $\mathrm{JH}$ in preventing precocious metamorphosis is documented in some hemimetabolous and holometabolous insects [6, 41-43]. We studied the contribution of CBP (HAT, acetylation) and TSA (HDAC inhibitor) to JH action. TcA cell line, originated from the non-embryonic adult insect tissues, was used in the study. The cells were treated with dsmalE (dsRNA targeting E. coli maltase gene as control) or $d s C B P$ followed by exposure to JH III, TSA or DMSO (Table 1).

\section{TcA cell response to different treatments}

The response of TcA cells to different treatments [MD (dsmalE+ DMSO), MJ (dsmalE+ JHIII), MT (dsmalE+ TSA), CD (dsCBP+ DMSO), CJ $(d s C B P+\mathrm{JHIII})$, and $\mathrm{CT}(d s C B P+\mathrm{TSA})]$ was determined before the sequencing experiments by quantifying the expression of $C B P$ and $K r-h 1$ genes in all the treatments using RT-qPCR method [33]. Results showed more than 50\% reduction of $C B P$ mRNA levels after $d s C B P$ treatment. The $K r-h 1$ mRNA levels in control cells exposed to dsmalE increased after JH or TSA treatment when compared to their levels in DMSO treated cells. However, there was no increase in $K r-h 1$ mRNA in cells exposed to dsCBP and treated with $\mathrm{JH}$ or TSA indicating the requirement of $C B P$ for the cellular response to JH and TSA treatments (Fig. 1). These results suggest a possible role for $C B P$ and TSA in JH induction of $K r-h 1$ gene.

\section{RNA-seq and gene expression analysis}

To identify the impact of $C B P$ on gene expression after JH III or TSA treatment, we performed sequencing of RNA isolated from TcA cells exposed to dsmalE or dsCBP followed by treatment with $\mathrm{JH}$ or TSA. Illumina sequencing resulted in 90.28, 64.05, 86.01, 98.4, 100.32 and 94.04 million reads for MD (dsmalE+ DMSO), M) (dsmale+ JHIII), MT (dsmalE+ TSA), CD (dsCBP+ DMSO), CJ (dsCBP+ JHIII) and CT $(d s C B P+\mathrm{TSA})$ treatments respectively. After quality control measures, approximately $83-93 \%$ of reads from all treatments were mapped back to the exon regions of the T. castaneum genome (Table 2). Further details of RNA-seq statistics for each biological replicate are included in Additional file 1: Table S2. After RPKM based gene expression normalization, differential gene expression (DGE) analysis was performed using EDGE analysis software package of CLC workbench, and the overall gene expression patterns for each treatment compared to the control (MD) are represented as heatmaps (Fig. 2a-e). Comparing with the control (MD), the gene expression levels in different samples was normalized in a way that made all treatments comparable to each other. Differentially expressed $(p<0.01$ and \pm 2 -fold $\uparrow \downarrow)$ genes for each comparison (Table 1) are represented as red dots in the volcano plots (Fig. 2f-j).

After statistical analysis, 32 and 699 genes were upregulated after JH (MD vs. MJ) or TSA (MD vs. MT) treatment of control cells exposed to dsmalE. Similarly, 181 and 602 genes were significantly upregulated in TcA cells exposed to $d s C B P$ followed by treatment with $\mathrm{JH}$ (MD vs. $\mathrm{CJ}$ ) or TSA (MD vs. CT). Also, $C B P$ knockdown 


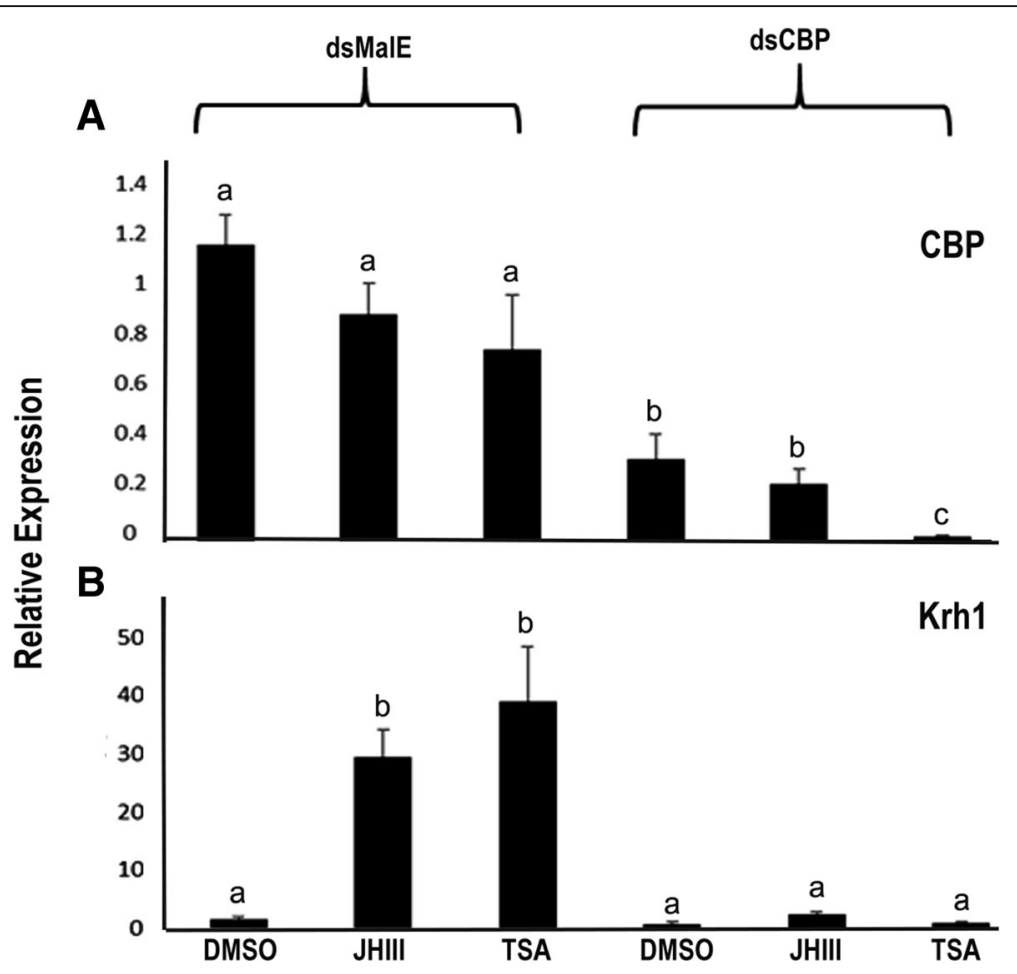

Fig. 1 TCA cells respond to both juvenile hormone and TSA after dsmalE and dsCBP treatment. Total RNA was isolated from 100,000 cells that were cultured in the medium containing either dsmalE or dsCBP for $72 \mathrm{~h}$. The cells were then exposed to DMSO $(<0.1 \%)$ or JH III $(2 \mu \mathrm{M})$ or TSA $(5 \mu \mathrm{M})$ for $6 \mathrm{~h}$. Total RNA was isolated from the treated cells and used to quantify mRNA levels of CBP (a) and Kr-h1 (b). The data shown are the mean + S.E. $(n=4)$. (Letters represent significance at $95 \% \mathrm{Cl})$

alone caused down-regulation of 456 genes in DMSO treated cells compared to control (MD vs. CD). Details on Blast2GO hits in the NR databases are included in Additional files 2, 3, 4, 5 and 6: Excel Files S1-S5. The expression of 15 selected genes from all the treatments was verified by RT-qPCR (Fig. 3). Comparison of the gene expression levels between RT-qPCR and RNA-seq study showed a correlation coefficient of 0.74 (Additional file 1 : Figure S1) indicating a good agreement of gene expression data obtained by both methods.

\section{$\mathrm{JH}$ response genes in TcA cells}

JH III treatment of TcA cells (dsmalE+ JHIII or MJ) caused an increase in expression of known $\mathrm{JH}$ response genes including $K r$-h1 (Fig. 4a), a mediator of anti-metamorphic action of JH [44-47] when compared to control cells treated with DMSO (MD or dsmalE+ DMSO). A decrease in JH titers at the end of final instar larval stage reduces the $K r-h 1$ mRNA levels that relax the inhibitory influence on metamorphic changes and leads to the onset of metamorphosis through an increase in expression of genes such as Broad and E93 required for pupal and adult development respectively $[8,48]$. In $T$. castaneum, RNAi-mediated depletion of $K r-h 1$ expression led to precocious pupation [45]. Besides $K r-h 1$, there were 31 other genes whose expression increased after $\mathrm{JH}$ treatment of TcA cells. Among them, eight genes including ankyrin 3, Vitellogenin (Vg), matrix metalloproteinase-3 (Mmp-3), neurexin-1 and alpha-2C adrenergic receptor showed expression pattern similar to $K r$-h1 in different comparisons (Fig. 4b). Ankyrin or ankyrin repeat containing proteins are involved in signal transduction processes in $D$. melanogaster and play a crucial role in the development or initiation of the immune response in eukaryotic cells [49-51]. Induction of ankyrin-3 gene by JH suggests its function in the regulation of gene expression in response to the immune challenge as reported previously [52]. Induction of $\mathrm{Vg}$ (a protein made by female insects to produce yolk) by JH in TcA cells support our previous finding on the regulation of $\mathrm{Vg}$ gene in T. castaneum by JH through insulin-like peptide signaling pathway [53]. In other insects including B. germanica, ectopic application of $\mathrm{JH}$ caused re-expression of $\mathrm{Vg}$ in $\mathrm{JH}$-deficient female adults [54]. Similarly, in Locusta migratoria, induction of $\mathrm{Vg}$ was observed after methoprene ( $\mathrm{JH}$ analog) injection [55].

Furthermore, $\mathrm{JH}$ also induced $M m p-3$, and it showed a similar expression profile as $K r$ - $h 1$ after various treatments. In T. castaneum, three Mmps, i.e., Mmp1, Mmp2, and $M m p 3$ were identified $[56,57]$. RNAi mediated knockdown of Mmp1 caused an arrest in the initial stage of pupation 


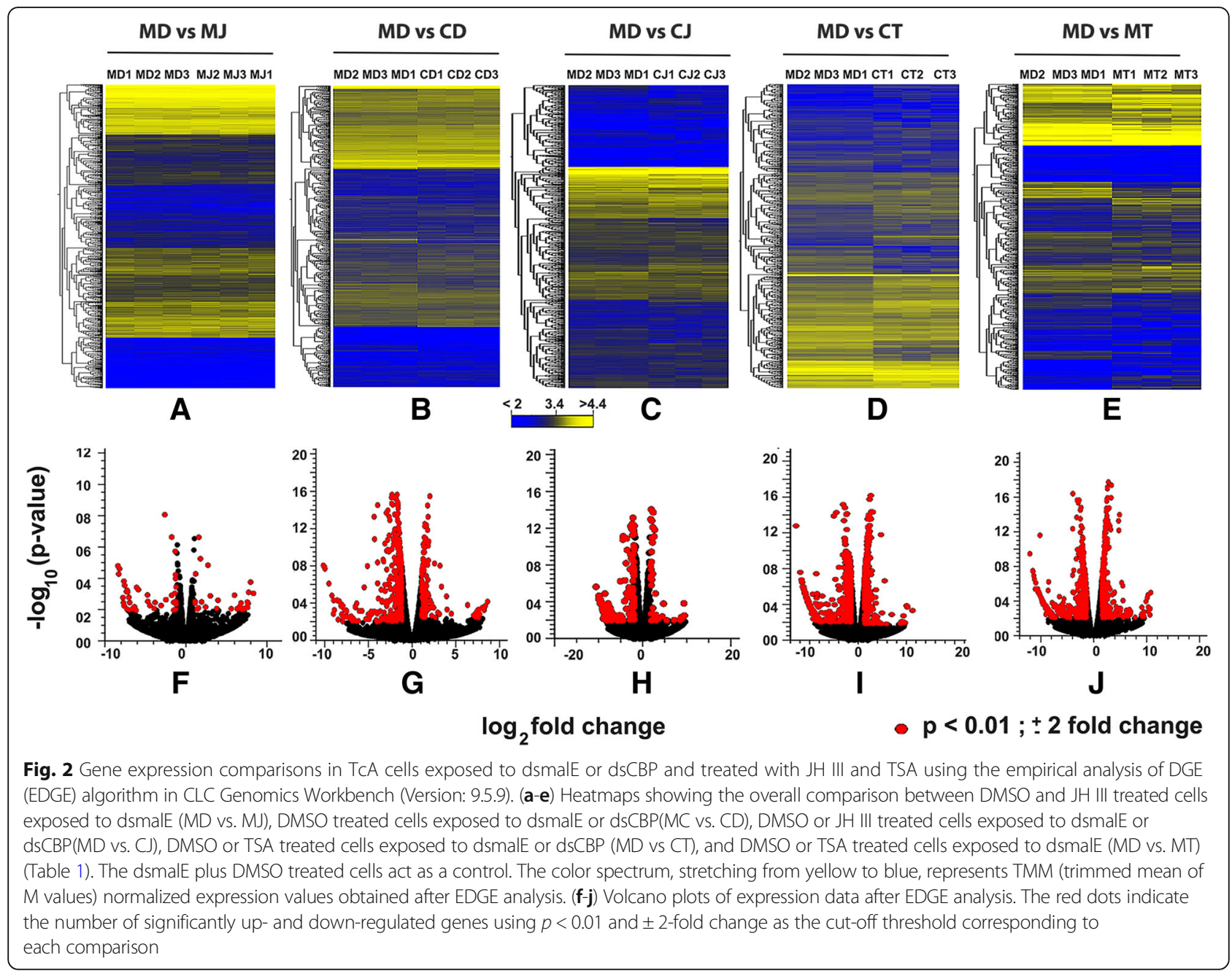

resulting in the altered development of wings, legs, antennae, compound eye, etc. Whereas, $M m p 2$ knockdown resulted in abnormal gut development during beetle embryogenesis [58]. However, RNAi mediated knockdown of Mmp3 did not show any abnormality although the expression of Mmp3 was highly upregulated during metamorphosis. Hence, it was postulated that both $M m p 2$ and $\mathrm{Mmp} 3 \mathrm{had}$ overlapping functions. Moreover, $\mathrm{JH}$ also induced an alpha $2 C$ adrenergic receptor, a G-protein coupled receptor (GPCR) in TcA cells. Interaction of GPCR with JH had been reported in other insects $[9,59]$. Our previous studies in $T$. castaneum screened multiple candidate GPCRs that may be involved in Vg uptake into oocytes and identified promising candidate membrane receptor mediating $\mathrm{JH}$ regulation of patency [11]. However, the contribution of intracellular and membrane receptors in $\mathrm{JH}$ action need to be investigated further.

\section{TSA-response genes}

Trichostatin A (TSA) is a class I and II histone deacetylase (HDACs) inhibitor. It is known that HDACs mediated epigenetic mechanisms regulate various physiological processes [60]. In the present study, TSA application resulted in up-regulation of approximately 699 genes in dsmalE treated cells (MT or dsmalE + TSA) when compared to control cells treated with DMSO (MD or dsmalE + DMSO) (Fig. 5a, Additional file 3: Excel File S2). 22\% of these genes contained "epifactor" domains (Fig. 5b) that are detected most frequently in proteins with capabilities of modifying histones and thus involved in chromatin remodeling [61]. Epifactor domains, observed predominantly within the gene set induced by TSA are shown in Fig. 5c. Some of these domains are well known due to their involvement in various cellular processes. For example, the SET domain proteins are involved in DNA methylation [62], and the Jmj (Jumonji) domain proteins are involved in histone demethylation [63]. It is known that 'eggless' family of SET domains fused to the TAM/ MBD domain play a distinct role in epigenetic regulation through DNA and histone methylation in Acyrthosiphon pisum [64]. In MD vs. MT comparison, we identified nine SET domain-containing genes that are induced by TSA 


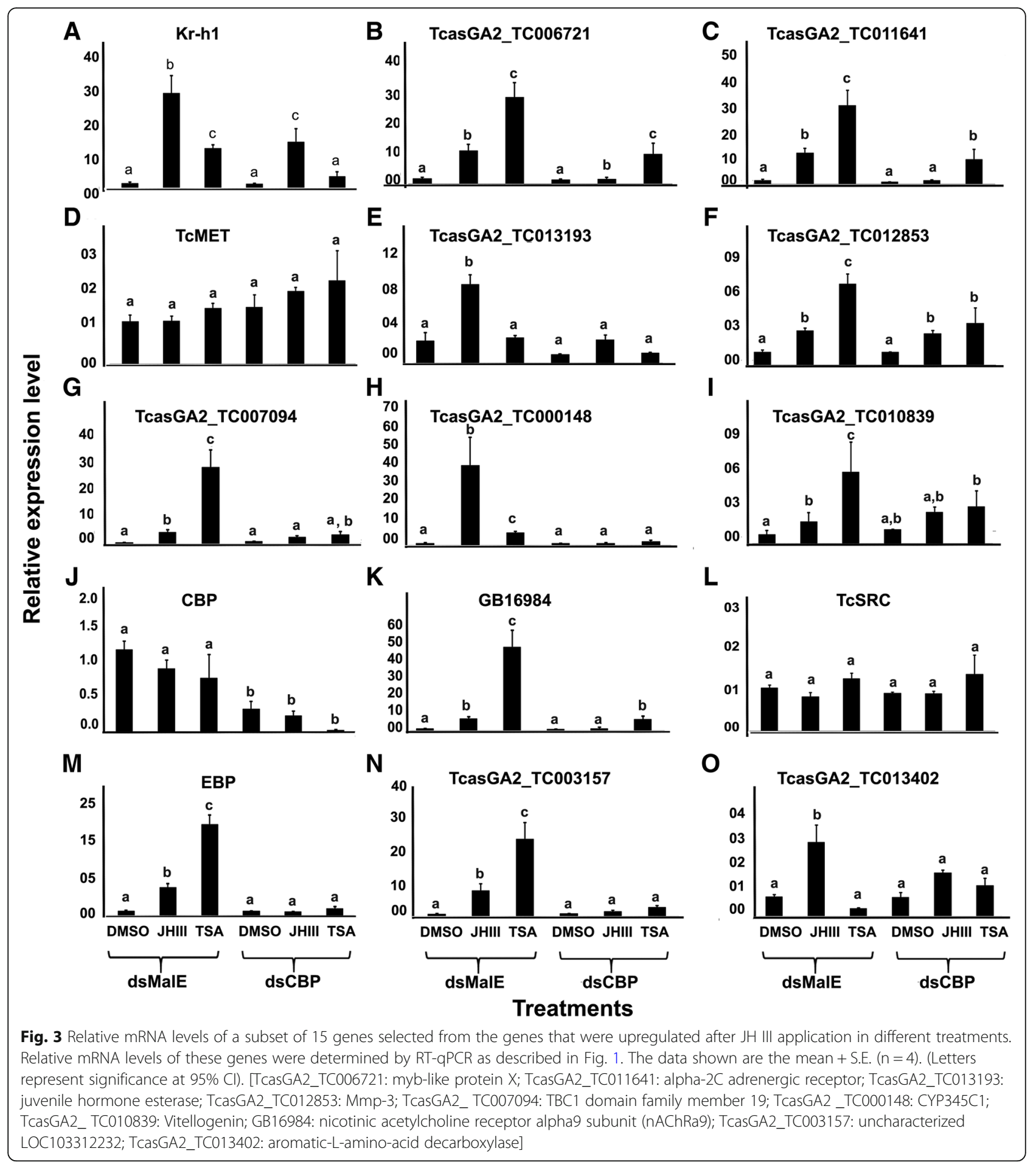

(Fig. 5c \& Additional file 3: Excel File S2). Moreover, genes coding for polycomb group protein Psc and trithorax that maintain active or repressed gene expression states in a cell by regulating chromatin architecture at multiple levels ranging from local structural modifications to the three-dimensional organization within the genome were also induced by TSA treatment. Indeed, most prominent epigenetic regulatory systems use these well-conserved groups of proteins to function antagonistically for orchestrating the expression of key genes during development [65].

Transcription factors are also important regulators of gene expression. In $D$. melanogaster, transcription factors containing helix-loop-helix, $\mathrm{C}_{2} \mathrm{H}_{2} \mathrm{Zn}$-finger or forkhead DNA-binding domain play essential roles in developmental 


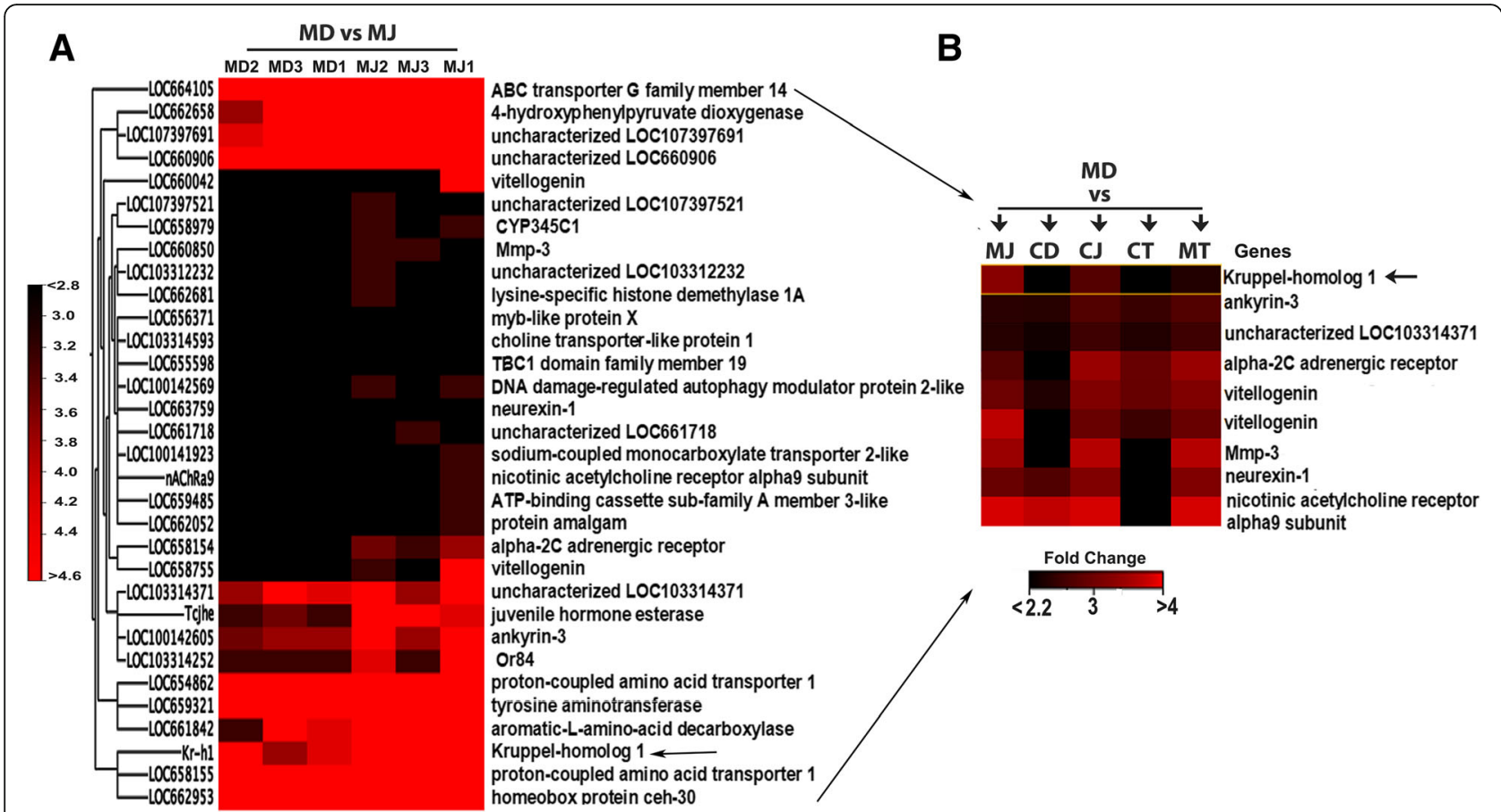

Fig. 4 Gene expression after JH III induction. (a) Heatmap illustrating the TMM normalized expression values of 32 up-regulated genes $(p<0.01$, $\leq 2$-fold $\uparrow$ ) after dsmalE and JH III treatment in TcA cells. (b) Heatmap showing the expression of genes in different treatments that are behaving similarly to $\mathrm{Kr}$-h1, the major JH-response gene. [MD: dsmalE (M) treated cells exposed to DMSO; MJ: dsmalE (M) treated cells exposed to JH III]

processes such as body plan determination and cell fate specification [66]. Interestingly, many genes coding for DNA-binding domain containing transcription factors were induced by TSA (Fig. 5c, Additional file 3: Excel File S2). A list of TSA response genes after various treatments is shown in Additional file 1: Figure S2. Interestingly, the expression levels of these genes decreased after $C B P$ knockdown suggesting that acetylation levels maintained by HATs $(C B P)$ and HDAC inhibitors (TSA) regulate the expression. Gene coding for Forkhead box protein $\mathrm{O}($ FoxO $)$, a transcription factor that plays important roles in the regulation of metabolism, development, and organogenesis through either activation or repression of the target gene expression, was induced by TSA (Additional file 3: Excel File S2). FoxO regulates lipolysis in fat body cells by regulating the expression of lipases (i.e., brummer, acid lipase-1) during molting and pupation in Bombyx mori [67]. Recently, Zeng et al., (2017) [68] documented the role of FoxO transcription factor in regulating JH degradation in Bombyx mori. However, the function of FoxO could vary among different insect orders. In D. melanogaster (Diptera), delayed metamorphosis was caused by overexpression of FoxO [69] whereas, RNAi of FoxO caused delayed pupation in T. castaneum (Coleoptera) [70]. FoxO also regulates the timing of pupation through regulating ecdysteroid biosynthesis in T. castaneum, [70]. Our previous studies also documented the regulation of $V g$ gene expression by FoxO and JH in T. castaneum [71].
Genes coding for nuclear receptors (NRs) including Ftz transcription factor 1 (Ftz-f1), Hormone receptor 51 (HR51), Seven-up (SVP) and Hormone receptor 38 (HR38) were induced by TSA. The NRs play a fundamental role in embryonic development and metamorphosis [72-75]. For example, expression of NRs, such as E75, HR3, and $F t z-f 1$ is crucial for the development of D. melanogaster embryos [76]. The NRs also serve as the early response genes in the 20E regulation of molting and metamorphosis in D. melanogaster [77]. During postembryonic development, the Ftz-f1 expression is required for successful molting and metamorphosis [78, 79]. Specifically, $\beta$ Ftz-f1 acts as a competence factor for stage-specific responses to $20 \mathrm{E}$ pulse that initiates the pupal molt [80]. Similarly, D. melanogaster HR38 regulates metamorphosis [81]. Although HR38 expression was not directly under the control of 20E, it participates in the 20E signaling pathway as an alternative partner of ultraspiracle (USP) [82]. Our previous RNAi-based studies demonstrated the role of TcFtz-f1 and TcHR51 during larval-pupal metamorphosis. Precisely, 100\% mortality before pupation was observed when $d s T c F t z-f 1$ and $d s T c H R 51$ were injected into one-day old final instar T. castaneum larvae [83]. Likewise, TcSVP and TcHR38 play crucial roles in both larval-pupal and pupal-adult metamorphosis as TcSVP, and TcHR38 RNAi larvae died either during larval-pupal transition or pupal-adult transition [83]. A similar RNAi-based study from our lab further disclosed the 
A

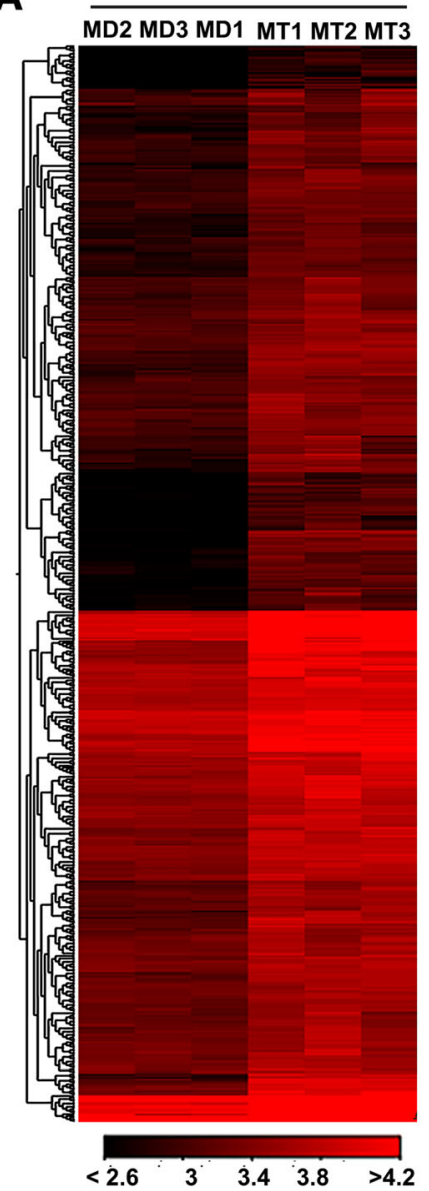

B
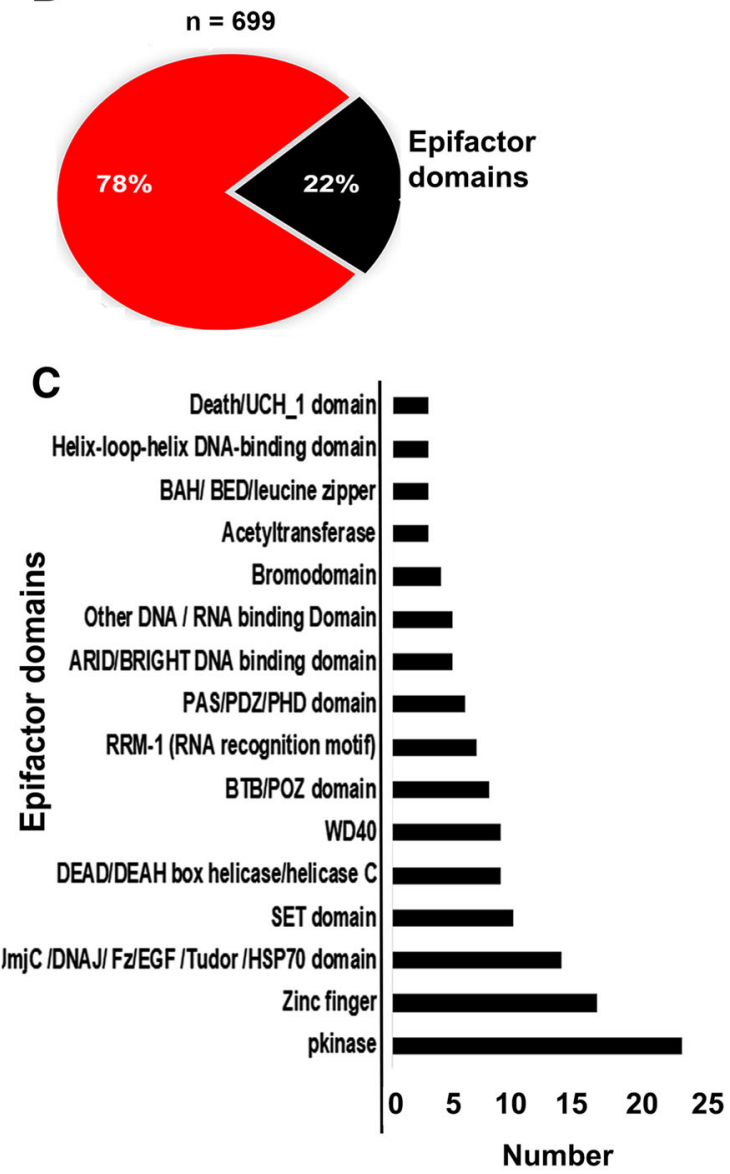

Fig. 5 Effect of TSA application on gene expression. (a) Heatmap illustrating the TMM normalized expression values of 699 up-regulated genes $(p<0.01, \geq 2$-fold $\uparrow)$ after TSA treatment in TcA cells. (b) Figure showing the percent containing epifactor domains within 699 genes. (c) Epifactor domains present in TSA induced genes. [MD: dsmalE treated cells exposed to DMSO; MT: dsmalE treated cells exposed to TSA]

importance of TcSVP in reproduction, as indicated by the observation that newly eclosed $T$. castaneum females injected with dsTcSVP caused a decrease in $T c V g$ transcript levels and egg production [84]. Recently, the contribution of SVP and Ftz-f1 in JH biosynthesis was also documented in B. germanica [85].

Interestingly, JH signal transduction pathway genes including $S R C$ and $K r-h 1$ were also induced by TSA. However, $S R C$ induction by TSA was not confirmed by RT-qPCR (Fig. 3). $K r-h 1$, a mediator of JH action during metamorphosis in most of the insects, was induced TSA. Remarkably, knockdown of CBP suppressed TSA induction of $K r-h 1$ (Additional file 3: Excel File S2). The expression of Met gene was unchanged by TSA suggesting some degree of selectivity (Fig. 3). The present study also revealed that TSA induces fatty acid synthase (FAS) expression. The contribution of FAS to lipid accumulation during diapause preparation in insects has been reported. Recently, Tan et al., [86] found that fatty acid synthase 2 contributed explicitly to lipid accumulation and stress tolerance gene expression in the beetle, Colaphellus. bowringi. Furthermore, the induction of calcium channel flower (LOC6 58279), calcium-transporting ATPase type 2C member 1 (LOC658547), calpain-C (LOC662931), etc. after TSA application suggested the possibility of regulation of gene expression through altering cellular $\mathrm{Ca}^{2+}$ homeostasis. However, such possibilities need to be confirmed.

The widespread changes in gene expression after TSA treatment was also shown in the pathway enrichment analysis of 699 genes induced by TSA. Some metabolic pathways including MAPK, Hippo, FoxO, and Wnt signaling and fatty acid metabolism, Longevity regulating pathways and TGF $\beta$ signaling pathway were also enriched after TSA treatment (Additional file 1: Figure S3A). Among them, the TGF- $\beta$ signaling pathway and FoxO signaling were shown to regulate insect metamorphosis [70, 87]. Thus TSA (alternatively HDACs) may indirectly involve in hormonal regulation during metamorphosis in T. castaneum. 


\section{CBP regulates genes involved in the development and hormone action}

CBP is a transcriptional co-activator with HAT activity regulating the activity of hundreds of transcription factors. During postembryonic development, Nejire (a homolog of $\mathrm{CBP}$ ), contributes to the ecdysone signaling pathway. Nejire regulates the expression of sox14, an ecdysone-response gene through acetylation of H3K27 [88]. Our previous in vivo studies in T. castaneum larvae indicated the role of CBP in insect postembryonic development [89]. However, due to interference from endogenous hormones, we did not obtain a satisfactory picture of the impact of $C B P$ RNAi on epigenetic regulation of $\mathrm{JH}$ action. In the current study, we aimed to get a more comprehensive picture using $\mathrm{JH}$ and TSA treatments after $C B P$ RNAi (Table 1). CBP knockdown (CD or $d s C B P+\mathrm{DMSO}$ ) caused down-regulation of 456 genes in TcA cells compared to control cells exposed to dsmalE (MD or dsmalE + DMSO) (Fig. 6a, Additional file 4: Excel S3). Nearly one third (approximately 147) of the 456 genes were also found to be down-regulated in the $C B P$ dsRNA injected T. castaneum larvae [89] (Fig. 6b, Additional file 7: Excel S6). Among these 147 genes, many of them such as FoxO, fatty acid synthase, hairy $(H)$, insulin receptor (InR), Laminin subunit alpha (LanA), adenosine deaminase, bambi, eyegone, etc. are known to be involved in hormonal regulation of development and metamorphosis. The contributions of FoxO and fatty acid synthase in hormone biosynthesis (20E) in the regulation of metamorphosis, reproduction, and lipid biosynthesis during diapause preparation were already discussed in the previous section. Among the other genes, JH-response gene hairy $(H)$ acts downstream to the Met in the $\mathrm{JH}$ action cascade in Aedes aegypti [90].

Insulin-like growth factor (IGF) signaling plays a vital role in insect growth, development and metabolism by controlling glucose uptake and protein translation. In $T$. castaneum, the two InRs play distinct roles during development and reproduction. Thus, down-regulation of $\operatorname{In} R$ by $C B P$ knockdown could affect those processes, but such an assumption needs to be experimentally confirmed. $\operatorname{Lan} A$ was another essential gene whose expression was affected by depletion of $C B P$ both in larvae and in TcA cells. Loss of function mutations of lanA gene in D. melanogaster caused embryonic lethality, whereas individuals with holomorphic alleles in specific combination gave rise to escaper adults with reduced viability and tissue defects demonstrating the contribution of LanA during D. melanogaster development [91]. Adenosine deaminase CECR1-A (Cat Eye Critical Region 1) expression was also affected by $C B P$ knockdown. In $D$. melanogaster, expression of adenosine deaminases increases during late larval stage suggesting that it may function during metamorphosis. The enzyme, adenosine deaminase may protect the tissues from toxic nucleosides released from cellular apoptosis during metamorphosis by transforming them into their respective inosine nucleosides and thus helping in tissue reconstruction during metamorphosis [92]. Reduced expression of these physiologically important genes by $C B P$ RNAi could induce mortality during metamorphosis as reported recently [89].

Transcription factors such as mad2 and bambi were also down-regulated in cells exposed to $d s C B P$. Bambi, a Bone Morphogenetic Protein (BMP) inhibitor present in T. castaneum and Apis genomes but not in Drosophila, was reported to be expressed in regions with high BMP signaling indicating its role in modulating TGF $\beta$ signaling [93]. In the dipteran model insect, D. melanogaster, TGF- $\beta$ signaling induced $\mathrm{JH}$ production through up-regulation of $J H$ acid methyltransferase (JHAMT) gene transcription through transcription factor Mothers Against Dpp (MAD) [94]. These data suggest that CBP could modulate the TGF $\beta$ signaling and $\mathrm{JH}$ biosynthesis by controlling the expression of bambi and JHAMT (via mad2). Similarly, the expression of Pax transcription factor gene eyegone (eyg) was also affected by depletion of CBP. In T. castaneum, eyegone is essential for normal development of hind wings, antennae, and eyes [95].

Some other key functional genes are affected in TcA cells after $C B P$ RNAi treatment. For instance, a nuclear receptor, $H R 38$, was down-regulated after $C B P$ RNAi in these cells. Besides $H 38$, the expression of other essential genes such as hexokinase 2 (HK2), Takeout, H17 and different cytochromes (i.e., Cyt18A1, Cyt303A1, Cyp4q6, $C y p 4 C 1$, etc.) that are known to be involved in various physiological function in insects, were affected by $C B P$ RNAi. HK2 was one of the key components in insect chitin biosynthesis pathway that contributes to insect growth and development [96, 97]. In T. castaneum, RNAi of trehalose- 6-phosphate synthase (TcTPS) significantly reduced the expression of $\mathrm{HK} 2$, and the larvae could not complete the transition from larvae to pupae due to a lower level of chitin and resulted in abnormal phenotypes [98]. Hence, down-regulation of $H K 2$ could also lead to abnormal molting phenotype by negatively influencing chitin synthesis in $T$. castaneum as observed in our recent study where $C B P$ was knocked down in $T$. castaneum larvae [89]. Similarly, the expression of takeout gene, reported to be regulated by $\mathrm{JH}$ [99], was also affected by $C B P$ RNAi. Interestingly, in $N$. lugens, the expression of the takeout gene increased after knockdown of $M e t$ and $K r-h 1$ genes and decreased by RNAi of Met interacting partners such as NITai and NI $\beta$-Ftz-f1 [99]. Although the depletion of $C B P$ has no direct effect on Met expression pattern, the indirect involvement of CBP via takeout in $\mathrm{JH}$ signaling pathway mediated by Met and other Met interacting partners is possible but needs to be experimentally validated. 

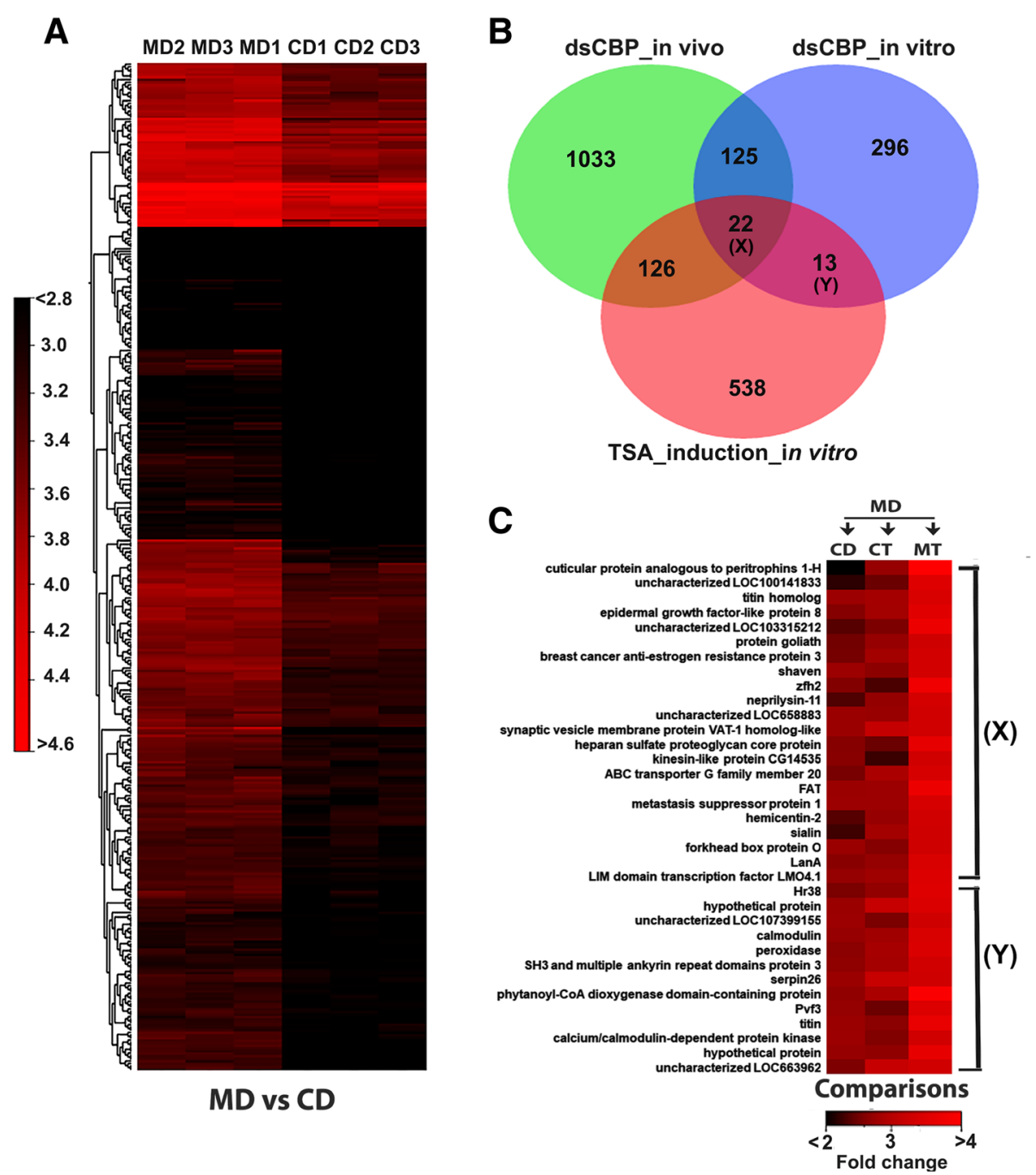

Fig. 6 Impact of dsCBP on gene expression. (a) Heatmap illustrating the TMM normalized expression values of 456 down-regulated genes $(p<0.01, \leq$ 2-fold $\downarrow$ ) after dsCBP treatment in TcA cells. (b) Venn diagram representing the genes that were commonly affected by dsCBP treatment in vitro, in vivo (data not shown), and induced after TSA treatment. Twenty-two genes $(\mathbf{X})$ were common in between all the comparisons (MD vs. CD, MD vs. MT, dsCBP in vivo) whereas thirteen genes (Y) were common in between comparisons such as MD vs. CD and MD vs. MT. (c) Heatmap showing the expression of selected genes that were represented as ' $X$ and ' $Y$ ' in section (b) after treatments of interest. [MD: dsmalE treated cells exposed to DMSO; CD: dsCBP treated cells exposed to DMSO; MT: dsmalE treated cells exposed to TSA]

Two genes, H17 and farnesol dehydrogenase, which play antagonistic roles in $\mathrm{JH}$ biosynthesis, were also affected by CBP RNAi. H17, a mimic of the allatostatin A, inhibits JH biosynthesis in B. germanica and thus acts as an insect growth regulator (IGR) [100]. The influence of allatostatin A on vitellogenesis was also documented in B. germanica [101]. CBP RNAi also affected farnesol dehydrogenase expression. Oxidation of NADP-dependent farnesol dehydrogenase is a rate-limiting step in $\mathrm{JH}$ biosynthesis pathway in adult mosquitoes [102]. The decrease in farnesol dehydrogenase expression could lead to reduced endogenous $\mathrm{JH}$ synthesis. Hence, CBP may also influence $\mathrm{JH}$ production in insects. Another gene family, Cytochrome P450s (CYPs), perform vital physiological functions during all life stages of an insect through their involvement in the biosynthesis of $20 \mathrm{E}$ and $\mathrm{JH}[103,104]$. The expression of different cytochrome P450 family members was downregulated by $C B P$ RNAi. For example, the expression of CYP18A1 that is essential for D. melanogaster growth and development was reduced after $C B P$ RNAi [105]. Some of the genes from a CYP4 family that is known to be involved in the metabolism of JH III were also downregulated by $C B P$ knockdown [106]. Furthermore, RNAi of $C B P$ decreased the expression of transcripts such as calcium-activated chloride channel regulator 4 (LOC662351), sodium/potassium-transporting ATPase subunit alpha (LOC663833) and calcium release-activated calcium channel protein 1(LOC657890) 
possibly suggesting a role in the maintenance of cellular $\mathrm{Ca}^{2+}$ homeostasis. However, additional experiments are needed to test this hypothesis.

Finally, Pathway enrichment analysis of the 456 downregulated genes revealed the signaling pathways regulated by CBP (Additional file 1: Figure S3 B). There were many pathways, related to insect growth, immunity and development, affected by $C B P$ RNAi. For instance, FoxO signaling, fatty acid metabolism, Insect hormone biosynthesis, Toll and IMD pathway, TGF- $\beta$, and Hedgehog signaling pathways were among the pathways enriched > 1.5 times in CBP RNAi samples. Functionally, FoxO signaling, insect hormone biosynthesis, and TGF- $\beta$ signaling are involved in insect hormone biosynthesis; reproduction and metamorphosis, Toll and IMD pathway are involved in insect immunity development and hedgehog signaling pathway involved in adult appendages patterning [87, 107-111].

Functional correlation between CBP RNAi and TSA treatment Both CBP and TSA work as epigenetic modulators of gene expression through their different roles as writer and eraser of acetylation. The expression of some genes was regulated by both CBP and TSA. We identified 35 genes that were regulated by both CBP and TSA (Fig. 6b, Additional file 7: Excel S6). The application of TSA after $C B P$ knockdown could not rescue the expression of these genes. Importantly, FoxO and HR38 that are known to mediate $\mathrm{JH}$ and 20E signaling respectively were affected by both CBP and TSA. These data suggest that acetylation (by HATs, e.g., CBP) and deacetylation (HDACs, TSA studies) could regulate expression of key players involved in $\mathrm{JH}$ and $20 \mathrm{E}$ action thus adding an extra layer of epigenetic regulation to hormone action (Fig. 6c, Additional file 7: Excel File S6). Pathway enrichment analysis has also revealed the regulation of common pathways by both CBP and TSA. Pathways such as TGF- $\beta$, FoxO, and Wnt signaling, ECM receptor interaction and fatty acid biosynthesis were regulated by both CBP and TSA in TcA cells. Interestingly, comparing the CBP RNAi in $T$. castaneum in larvae [89] and TSA treatment of TcA cells, we found 148 genes that were affected by both these treatments. Among them, Kr-h1, svp, Fz2, polycomb, lanA, FoxO and $B M P$ receptor type- $1 B$ were shown to regulate insect development and metamorphosis (Additional file 7: Excel S6). Moreover, $\sim 22$ genes including FoxO and $\operatorname{lan} A$ responded to $C B P$ RNAi in larvae and TcA cells as well as TSA treatment in TcA cells (Fig. $6 \mathrm{~b}$ and c; Additional file 7: excel S6). The expression levels of FoxO was further validated by RT-qPCR (Additional file 1: Figure S4). The function of FoxO in T. castaneum growth, reproduction and metamorphosis has been reported previously, but the report on epigenetic regulation of FoxO through CBP and HDACs is one of the major contributions of this paper.
However, the effect of epigenetic regulation of $F o x O$ on hormone action requires further studies.

\section{Conclusions}

$\mathrm{JH}$ and 20E play vital roles in insect life by orchestrating the molting and metamorphosis processes. Synthesis of these hormones and their spatiotemporal action are indispensable for successful molting and metamorphosis. Such level of precision in the synthesis of these hormones and expression of their downstream response genes during different life stages of insects indeed demands a higher degree of transcriptional as well as translational regulation. The present study shows the function of CBP and HDAC in regulating JH hormone action and corroborates our previous findings on T. castaneum larvae with only $C B P$ RNAi treatment. The impact of $d s C B P$ and TSA on the expression of the $\mathrm{JH}$ response gene, $K r-h 1$, was confirmed. The expression of $V g$ gene was also affected by $d s C B P$ to such an extent that the expression level did not recover by further $\mathrm{JH}$ treatment. The expression of other key developmental genes such as bambi, eyegone, FoxO, lanA, svp, polycomb, Fz-2, HR38 and hairy was also influenced by the CBP RNAi and/or HDAC inhibition. Many key physiological pathways involved in insect growth and development such as TGF- $\beta$ and FoxO signaling were modulated by both treatments. Thus, CBP and HDAC may contribute significantly to the hormonal regulation of $T$. castaneum metamorphosis. Interestingly, FoxO gene expression was significantly altered after $C B P$ RNAi or TSA treatment confirming the epigenetic regulation of this important transcription factor. Further studies based on chip sequencing and functional proteomics will help to elucidate the precise mechanisms involved in epigenetic regulation of $\mathrm{JH}$ action in insects.

\section{Additional files}

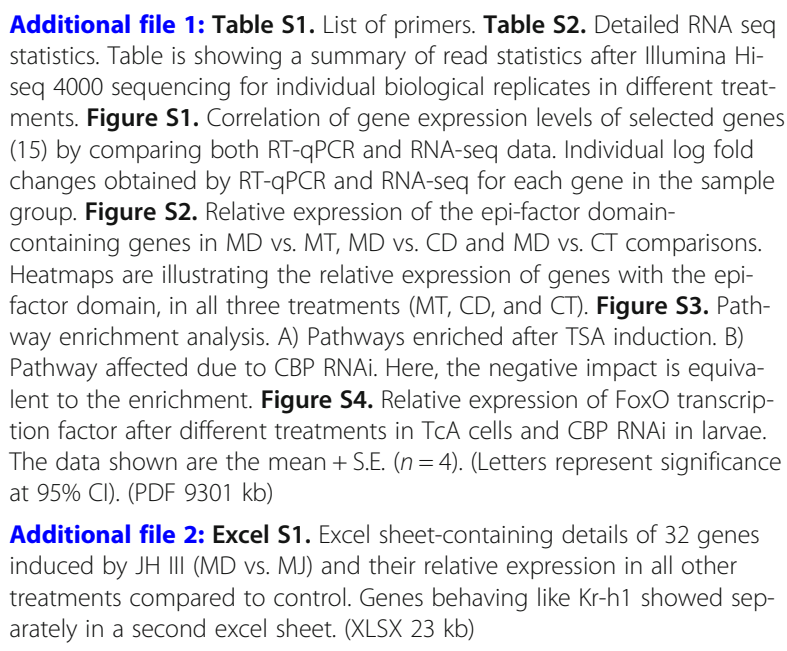
statistics. Table is showing a summary of read statistics after Illumina Hiseq 4000 sequencing for individual biological replicates in different treatments. Figure S1. Correlation of gene expression levels of selected genes (15) by comparing both RT-qPCR and RNA-seq data. Individual log fold changes obtained by RT-qPCR and RNA-seq for each gene in the sample group. Figure S2. Relative expression of the epi-factor domaincontaining genes in MD vs. MT, MD vs. CD and MD vs. CT comparisons. Heatmaps are illustrating the relative expression of genes with the epifactor domain, in all three treatments (MT, CD, and CT). Figure S3. Pathway enrichment analysis. A) Pathways enriched after TSA induction. B) Pathway affected due to CBP RNAi. Here, the negative impact is equivalent to the enrichment. Figure S4. Relative expression of FoxO transcription factor after different treatments in TcA cells and CBP RNAi in larvae. The data shown are the mean + S.E. $(n=4)$. (Letters represent significance at $95 \% \mathrm{Cl}$ ). (PDF $9301 \mathrm{~kb}$ )

Additional file 2: Excel S1. Excel sheet-containing details of 32 genes induced by $\mathrm{JH}$ III (MD vs. MJ) and their relative expression in all other treatments compared to control. Genes behaving like Kr-h1 showed separately in a second excel sheet. (XLSX $23 \mathrm{~kb}$ ) 
Additional file 3: Excel S2. Excel sheet containing details of 699 genes induced by TSA (MD vs. MT) and their relative expression in other treatments of interest such as MD vs. CD and MD vs. CT. Genes with epifactor domains showed separately in a second excel sheet. Expression of Met and SRC in all three treatments showed in another excel sheet. (XLSX $133 \mathrm{~kb})$

Additional file 4: Excel S3. Excel sheet containing details of 456 genes suppressed by CBP RNAi (MD vs. CD) and their relative expression in all other comparisons such as MD vs. CT, MD vs. MJ, MD vs. CJ and MD vs. MT. (XLSX $89 \mathrm{~kb})$

Additional file 5: Excel S4. Excel sheet containing details of 181 genes responded after CBP RNAi and subsequent JH III treatment. (XLS $69 \mathrm{~kb}$ )

Additional file 6: Excel S5. Excel sheet containing details of 602 genes responded after CBP RNAi and subsequent TSA treatment. (XLS $132 \mathrm{~kb}$ )

Additional file 7: Excel S6. Excel sheet containing details of gene clusters obtained after Venn diagram analysis shown in main Fig. 6b. (XLSX $43 \mathrm{~kb})$

\section{Abbreviations}

20E: 20-hydroxyecdysone; CD: dsCBP treated cells exposed to DMSO; CJ: dsCBP treated cells exposed to $\mathrm{JH} I I l ; C T$ : dsCBP treated cells exposed to TSA; dsCBP: dsRNA targeting a fragment of CREB-binding protein; dsmalE: dsRNA targeting a fragment of E. coli maltase gene; EcR: Ecdysone receptor; FOXO: Forkhead transcription factor; GPCR: G-protein-coupled receptor; HATs: Histone acetyltransferases; HDACs: Histone deacetylases; JHAMT: JH acid methyltransferase; JHIII: Juvenile Hormone III; Kr-h1: Krüppel homolog 1; MD: dsmalE treated cells exposed to DMSO; Met: Methoprene-tolerant; MJ: dsmalE treated cells exposed to JH III; Mmp-3: Matrix metalloproteinase-3; MT: dsmalE treated cells exposed to TSA; nAChRa9: Nicotinic acetylcholine receptor alpha9 subunit; SRC: Steroid receptor coactivator; TcA cells: Tribolium castaneum cells; TSA: Trichostatin A

\section{Acknowledgments}

This is publication number 18-08-096 from the Kentucky Agricultural Experimental Station and published with the approval of the director.

\section{Funding}

This work is supported by a grant from the National Institutes of Health (GM070559-11) and the National Institute of Food and Agriculture, USDA, HATCH under 2351177000. AR is supported by EXTEMIT - K, No. CZ. 02. 1.01/ 0.0/0.0/15_003/0000433 financed by OP RDE during the preparation of part of the manuscript.

\section{Availability of data and materials}

We have deposited the short read (IIlumina HiSeq4000) data with the following accession number: PRJNA392465 [EBI short read archive (SRA)], SRA Ids - SRR5787268 to SRR5787285. The complete study can also be accessed directly at the following URL: http:// www.ebi.ac.uk /ena/data /view/ PRJNA392465.

\section{Authors' contributions}

AR and SRP designed research, AR conducted the experiments, AR and SRP wrote the manuscript, and both authors approved the manuscript.

\section{Ethics approval and consent to participate}

Not applicable.

\section{Consent for publication}

Not applicable.

\section{Competing interests}

The authors declare that they have no competing interests.

\section{Publisher's Note}

Springer Nature remains neutral with regard to jurisdictional claims in published maps and institutional affiliations.

\section{Author details}

'Department of Entomology, College of Agriculture, Food and Environment, University of Kentucky, Lexington, KY 40546, USA. Faculty of Forestry and Wood Sciences, EXTEMIT-K, Czech University of Life Sciences, Kamýcká 1176, Prague 6, 16521 Suchdol, Czech Republic.

Received: 11 April 2018 Accepted: 28 November 2018

Published online: 14 December 2018

\section{References}

1. Buszczak M, Segraves WA. Insect metamorphosis: out with the old, in with the new. Curr Biol. 2000;10(22):R830-3.

2. Truman JW, Riddiford LM. Endocrine insights into the evolution of metamorphosis in insects. Annu Rev Entomol. 2002;47(1):467-500.

3. Yamanaka N, Rewitz KF, O'Connor MB. Ecdysone control of developmental transitions: lessons from Drosophila research. Annu Rev Entomol. 2013;58: 497-516.

4. Dubrovsky EB. Hormonal cross talk in insect development. Trends Endocrinol Metab. 2005;16(1):6-11.

5. Nakagawa $Y$, Henrich VC. Arthropod nuclear receptors and their role in molting. FEBS J. 2009;276(21):6128-57.

6. Riddiford LM. How does juvenile hormone control insect metamorphosis and reproduction? Gen Comp Endocrinol. 2012;179(3):477-84.

7. Jindra M, Palli SR, Riddiford LM. The juvenile hormone signaling pathway in insect development. Annu Rev Entomol. 2013;58:181-204.

8. Kayukawa T, Jouraku A, Ito Y, Shinoda T. Molecular mechanism underlying juvenile hormone-mediated repression of precocious larval-adult metamorphosis. Proc Natl Acad Sci U S A. 2017;201615423.

9. Liu P, Peng H-J, Zhu J. Juvenile hormone-activated phospholipase C pathway enhances transcriptional activation by the methoprene-tolerant protein. Proc Natl Acad Sci U S A. 2015;112(15):E1871-9.

10. Li K, Jia QQ, Li S. Juvenile hormone signaling-a mini review. Insect science. 2018.

11. Bai $H$, Palli SR. Identification of $G$ protein-coupled receptors required for vitellogenin uptake into the oocytes of the red flour beetle, Tribolium castaneum. Sci Rep. 2016:6:27648.

12. Cai M-J, Dong D-J, Wang Y, Liu P-C, Liu W, Wang J-X, Zhao X-F. G-proteincoupled receptor participates in 20-hydroxyecdysone signaling on the plasma membrane. Cell Communication and Signaling. 2014;12(1):9.

13. Luft UC, Bychkov R, Gollasch M, Gross V, Roullet J-B, McCarron DA, Ried C, Hofmann F, Yagil Y, Yagil C. Farnesol blocks the L-type Ca2+ channel by targeting the a1C subunit. Arterioscler Thromb Vasc Biol. 1999:19(4):959-66.

14. Wyatt GR, Davey KG. Cellular and molecular actions of juvenile hormone. II. Roles of juvenile hormone in adult insects. Adv Insect Physiol. 1996;26:1-155.

15. Soller M, Bownes M, Kubli E. Control of oocyte maturation in sexually matureDrosophilafemales. Dev Biol. 1999;208(2):337-51.

16. Herndon L, Chapman T, Kalb J, Lewin S, Partridge L, Wolfner M. Mating and hormonal triggers regulate accessory gland gene expression in male Drosophila. J Insect Physiol. 1997;43(12):1117-23.

17. Belgacem $\mathrm{YH}$, Martin J-R. Neuroendocrine control of a sexually dimorphic behavior by a few neurons of the pars intercerebralis in Drosophila. Proc Natl Acad Sci U S A. 2002;99(23):15154-8.

18. Parthasarathy R, Tan A, Sun Z, Chen Z, Rankin M, Palli SR. Juvenile hormone regulation of male accessory gland activity in the red flour beetle, Tribolium castaneum. Mech Dev. 2009:126(7):563-79.

19. Akimaru H, Chen Y, Dai P, Hou D-X, Nonaka M, Smolik SM, Armstrong S, Goodman RH, Ishii S. Drosophila CBP is a co-activator of cubitus interruptus in hedgehog signalling. Nature. 1997;386(6626):735-8.

20. Akimaru H, Hou D-X, Ishii S. Drosophila CBP is required for dorsaldependent twist gene expression. Nat Genet. 1997;17(2):211-4.

21. Hung H-C, Maurer C, Kay SA, Weber F. Circadian transcription depends on limiting amounts of the transcription co-activator nejire/CBP. J Biol Chem. 2007;282(43):31349-57.

22. McManus KJ, Hendzel MJ. CBP, a transcriptional coactivator and acetyltransferase. Biochem Cell Biol. 2001;79(3):253-66.

23. Goodman RH, Smolik S. CBP/p300 in cell growth, transformation, and development. Genes Dev. 2000;14(13):1553-77.

24. Ylla G, Belles X. Corrigendum to "towards understanding the molecular basis of cockroach tergal gland morphogenesis. A transcriptomic approach"[insect Biochem. Mol. Biol. 63 (2015) 104-112]. Insect Biochem Mol Biol. 2015;65:108. 
25. Spannhoff A, Kim YK, Raynal NJM, Gharibyan V, Su MB, Zhou YY, Li J, Castellano S, Sbardella G, Issa JPJ. Histone deacetylase inhibitor activity in royal jelly might facilitate caste switching in bees. EMBO Rep. 2011;12(3):238-43.

26. Lockett GA, Wilkes F, Helliwell P, Maleszka R. Contrasting effects of histone deacetylase inhibitors on reward and aversive olfactory memories in the honey bee. Insects. 2014;5(2):377-98.

27. Ozawa T, Mizuhara T, Arata M, Shimada M, Niimi T, Okada K, Okada Y, Ohta K. Histone deacetylases control module-specific phenotypic plasticity in beetle weapons. Proc Natl Acad Sci. 2016;113(52):15042-7.

28. Simola DF, Graham RJ, Brady CM, Enzmann BL, Desplan C, Ray A, Zwiebel $\sqcup$, Bonasio R, Reinberg D, Liebig J. Epigenetic (re) programming of castespecific behavior in the ant Camponotus floridanus. Science. 2016;351(6268): aac6633.

29. Kayukawa T, Tateishi $K$, Shinoda T. Establishment of a versatile cell line for juvenile hormone signaling analysis in Tribolium castaneum. Sci Rep. 2013;3:1570

30. Xu J, Roy A, Palli SR. CREB-binding protein plays key roles in juvenile hormone action in the red flour beetle, Tribolium Castaneum. Sci Rep. 2018:8(1):1426.

31. Goodman CL, Stanley D, Ringbauer JA, RW JB, Silver K, Park Y. A cell line derived from the red flour beetle Tribolium castaneum (Coleoptera: Tenebrionidae). In vitro cellular \& developmental biology Animal. 2012;48(7):426-33.

32. Zhang Z, Xu J, Sheng Z, Sui Y, Palli SR. Steroid receptor co-activator is required for juvenile hormone signal transduction through a bHLH-PAS transcription factor, methoprene tolerant. J Biol Chem. 2011;286(10):8437-47.

33. Kalsi M, Palli SR. Cap n collar transcription factor regulates multiple genes coding for proteins involved in insecticide detoxification in the red flour beetle, Tribolium castaneum. Insect Biochem Mol Biol. 2017;90:43-52.

34. Ma L, Pati PK, Liu M, Li QQ, Hunt AG. High throughput characterizations of poly (a) site choice in plants. Methods. 2014;67(1):74-83.

35. Richards S, Gibbs RA, Weinstock GM, Brown SJ, Denell R, Beeman RW, Gibbs R, Bucher G, Friedrich M, Grimmelikhuijzen CJ. The genome of the model beetle and pest Tribolium castaneum. Nature. 2008;452(7190):949-55.

36. Roy A, Walker WB, Vogel H, Kushwaha S, Chattington S, Larsson M, Anderson P, Heckel D, Schlyter F. Data set for diet specific differential gene expression analysis in three Spodoptera moths. Data in brief. 2016;8:448-55.

37. Kalsi M, Palli SR. Transcription factors, CncC and Maf, regulate expression of CYP6BQ genes responsible for deltamethrin resistance in Tribolium castaneum. Insect Biochem Mol Biol. 2015;65:47-56.

38. Parthasarathy R, Tan A, Palli SR. bHLH-PAS family transcription factor methoprene-tolerant plays a key role in $\mathrm{JH}$ action in preventing the premature development of adult structures during larval-pupal metamorphosis. Mech Dev. 2008;125:601-16.

39. Xu J, Sheng Z, Palli SR. Juvenile hormone and insulin regulate trehalose homeostasis in the red flour beetle, Tribolium castaneum. PLoS Genet. 2013, 9(6):e1003535

40. Nijhout HF. Insect Hormones. Princeton: Princeton Univ. Press; 1994.

41. Staal G. Anti juvenile hormone agents. Annu Rev Entomol. 1986;31(1):391-429.

42. Tan A, Tanaka H, Tamura T, Shiotsuki T. Precocious metamorphosis in transgenic silkworms overexpressing juvenile hormone esterase. Proc Natl Acad Sci U S A. 2005;102(33):11751-6.

43. Minakuchi C, Namiki T, Yoshiyama M, Shinoda T. RNAi-mediated knockdown of juvenile hormone acid O-methyltransferase gene causes precocious metamorphosis in the red flour beetle Tribolium castaneum. FEBS J. 2008; 275(11):2919-31.

44. Kayukawa T, Murata M, Kobayashi I, Muramatsu D, Okada C, Uchino K, Sezutsu H, Kiuchi M, Tamura T, Hiruma K. Hormonal regulation and developmental role of Krüppel homolog 1, a repressor of metamorphosis, in the silkworm Bombyx mori. Dev Biol. 2014;388(1):48-56.

45. Minakuchi C, Namiki T, Shinoda T. Kruppel homolog 1, an early juvenile hormone-response gene downstream of Methoprene-tolerant, mediates its anti-metamorphic action in the red flour beetle Tribolium castaneum. Dev Biol. 2009:325(2):341-50.

46. Minakuchi C, Zhou X, Riddiford LM. Krüppel homolog 1 (Kr-h1) mediates juvenile hormone action during metamorphosis of Drosophila melanogaster. Mech Dev. 2008;125(1):91-105.

47. Parthasarathy R, Tan A, Bai H, Palli SR. Transcription factor broad suppresses precocious development of adult structures during larval-pupal metamorphosis in the red flour beetle, Tribolium castaneum. Mech Dev. 2008;125(3-4):299-313.

48. Zhou B, Hiruma K, Shinoda T, Riddiford LM. Juvenile hormone prevents ecdysteroid-induced expression of broad complex RNAs in the epidermis of the tobacco hornworm, Manduca sexta. Dev Biol. 1998;203(2):233-44.
49. Dushay MS, Asling B, Hultmark D. Origins of immunity: relish, a compound Rel-like gene in the antibacterial defense of Drosophila. Proc Natl Acad Sc U S A. 1996;93(19):10343-7.

50. Mosavi LK, Cammett TJ, Desrosiers DC, Zy P. The ankyrin repeat as molecular architecture for protein recognition. Protein Sci. 2004;13(6):1435-48.

51. Voronin D, Kiseleva E. Functional role of proteins containing ankyrin repeats. Cell Tiss Biol. 2008;49(12):989-99.

52. Flatt T, Heyland A, Rus F, Porpiglia E, Sherlock C, Yamamoto R, Garbuzov A, Palli SR, Tatar M, Silverman N. Hormonal regulation of the humoral innate immune response in Drosophila melanogaster. J Exp Biol. 2008;211(16):2712-24.

53. Sheng Z, Xu J, Bai H, Zhu F, Palli SR. Juvenile hormone regulates vitellogenin gene expression through insulin-like peptide signaling pathway in the red flour beetle, Tribolium castaneum. J Biol Chem. 2011;286(49): 41924-36.

54. Comas D, Piulachs M-D, Bellés X. Induction of vitellogenin gene transcription in vitro by juvenile hormone in Blattella germanica. Mol Cell Endocrinol. 2001;183(1):93-100.

55. Chinzei $Y$, White $B$, Wyatt G. Vitellogenin mRNA in locust fat body: identification, isolation, and quantitative changes induced by juvenile hormone. Can J Biochem. 1982;60(3):243-51.

56. Llano E, Adam G, Pendás AM, Quesada V, Sánchez LM, Santamaría I, Noselli S, López-Otín C. Structural and enzymatic characterization of Drosophila Dm2-MMP, a membrane-bound matrix metalloproteinase with tissuespecific expression. J Biol Chem. 2002;277(26):23321-9.

57. Llano E, Pendás AM, Aza-Blanc P, Kornberg TB, López-Otín C. Dm1-MMP, a matrix metalloproteinase fromDrosophila with a potential role in extracellular matrix remodeling during neural development. J Biol Chem. 2000;275(46):35978-85.

58. Knorr E, Schmidtberg H, Vilcinskas A, Altincicek B. MMPs regulate both development and immunity in the Tribolium model insect. PLoS One. 2009; 4(3):e4751.

59. Sevala VL, Davey K, Prestwich GD. Photoaffinity labeling and characterization of a juvenile hormone binding protein in the membranes of follicle cells of Locusta migratoria. Insect Biochem Mol Biol. 1995;25(2):267-73.

60. Xiao W, Chen X, Liu X, Luo L, Ye S, Liu Y. Trichostatin a, a histone deacetylase inhibitor, suppresses proliferation and epithelial-mesenchymal transition in retinal pigment epithelium cells. J Cellular Mol Med. 2014;18(4): 646-55.

61. Medvedeva YA, Lennartsson A, Ehsani R, Kulakovskiy IV, Vorontsov IE, Panahandeh P, Khimulya G, Kasukawa T, Consortium F, Drabløs F. EpiFactors: a comprehensive database of human epigenetic factors and complexes. Database. 2015;2015:bav067.

62. Dillon SC, Zhang X, Trievel RC, Cheng X. The SET-domain protein superfamily: protein lysine methyltransferases. Genome Biol. 2005;6(8):227.

63. Takeuchi T, Watanabe Y, Takano-Shimizu T, Kondo S. Roles of jumonji and jumonji family genes in chromatin regulation and development. Dev Dyn. 2006:235(9):2449-59.

64. Vidal NM, Grazziotin AL, lyer LM, Aravind L, Venancio TM. Transcription factors, chromatin proteins and the diversification of Hemiptera. Insect Biochem Mol Biol. 2016;69:1-13.

65. Schuettengruber B, Chourrout D, Vervoort M, Leblanc B, Cavalli G. Genome regulation by polycomb and trithorax proteins. Cell. 2007;128(4):735-45.

66. McGinnis W, Krumlauf R. Homeobox genes and axial patterning. Cell. 1992; 68(2):283-302

67. Hossain MS, Liu Y, Zhou S, Li K, Tian L, Li S. 20-Hydroxyecdysone-induced transcriptional activity of FoxO upregulates brummer and acid lipase-1 and promotes lipolysis in Bombyx fat body. Insect Biochem Mol Biol. 2013;43(9): 829-38

68. Zeng B, Huang Y, Xu J, Shiotsuki T, Bai H, Palli SR, Huang Y, Tan A. The FOXO transcription factor controls insect growth and development by regulating juvenile hormone degradation in the silkworm, Bombyx mori. J Biol Chem. 2017. https://doi.org/10.1074/jbc.M117.777797.

69. Koyama T, Rodrigues MA, Athanasiadis A, Shingleton AW, Mirth CK. Nutritional control of body size through FoxO-Ultraspiracle mediated ecdysone biosynthesis. Elife. 2014;3:e03091.

70. Lin X, Yu N, Smagghe G. FoxO mediates the timing of pupation through regulating ecdysteroid biosynthesis in the red flour beetle, Tribolium castaneum. In: Gen comp Endocrinol; 2017.

71. Parthasarathy R, Palli SR. Molecular analysis of nutritional and hormonal regulation of female reproduction in the red flour beetle, Tribolium castaneum. Insect Biochem Mol Biol. 2011;41(5):294-305. 
72. DeLuca HF. Overview of general physiologic features and functions of vitamin D. American J Clin Nutr. 2004;80(6):1689S-96S.

73. Martín D. Nuclear Receptor. In: Functions of nuclear receptors in insect development. Dordrecht, Heidelberg, London, New York: Springer; 2010. p. 31-61.

74. Fahrbach SE, Smagghe G, Velarde RA. Insect nuclear receptors. Annu Rev Entomol. 2012;57:83-106.

75. Jarvela AMC, Pick L. Chapter two-the function and evolution of nuclear receptors in insect embryonic development. Curr Top Dev Biol. 2017;125:39-70.

76. Carney GE, Wade AA, Sapra R, Goldstein ES, Bender M. DHR3, an ecdysoneinducible early-late gene encoding a Drosophila nuclear receptor, is required for embryogenesis. Proc Natl Acad Sci U S A. 1997;94(22):12024-9.

77. Sullivan AA, Thummel CS. Temporal profiles of nuclear receptor gene expression reveal coordinate transcriptional responses during Drosophila development. Mol Endocrinol. 2003;17(11):2125-37.

78. M-a Y, Murata T, Hirose S, Lavorgna G, Suzuki E, Ueda H. Temporally restricted expression of transcription factor betaFTZ-F1: significance for embryogenesis, molting and metamorphosis in Drosophila melanogaster. Development. 2000;127(23):5083-92.

79. Cruz J, Martín D, Bellés X. Redundant ecdysis regulatory functions of three nuclear receptor HR3 isoforms in the direct-developing insect Blattella germanica. Mech Dev. 2007;124(3):180-9.

80. Broadus J, McCabe JR, Endrizzi B, Thummel CS, Woodard CT. The Drosophila BFTZ-F1 orphan nuclear receptor provides competence for stage-specific responses to the steroid hormone ecdysone. Mol Cell. 1999;3(2):143-9.

81. Kozlova T, Pokholkova G, Tzertzinis G, Sutherland J, Zhimulev I, Kafatos F. Drosophila hormone receptor 38 functions in metamorphosis: a role in adult cuticle formation. Genetics. 1998;149(3):1465-75.

82. Baker KD, Shewchuk LM, Kozlova T, Makishima M, Hassell A, Wisely B, Caravella JA, Lambert MH, Reinking JL, Krause H. The Drosophila orphan nuclear receptor DHR38 mediates an atypical ecdysteroid signaling pathway. Cell. 2003;113(6):731-42.

83. Tan A, Palli SR. Identification and characterization of nuclear receptors from the red flour beetle, Tribolium castaneum. Insect Biochem Mol Biol. 2008; 38(4):430-9.

84. Xu J, Tan A, Palli SR. The function of nuclear receptors in regulation of female reproduction and embryogenesis in the red flour beetle, Tribolium castaneum. J Insect Physiol. 2010;56(10):1471-80.

85. Borras-Castells F, Nieva C, Maestro JL, Maestro O, Belles X, Martín D. Juvenile hormone biosynthesis in adult Blattella germanica requires nuclear receptors seven-up and FTZ-F1. Sci Rep. 2017;7:40234

86. Tan Q-Q, Liu W, Zhu F, Lei C-L, Wang X-P. Fatty acid synthase 2 contributes to diapause preparation in a beetle by regulating lipid accumulation and stress tolerance genes expression. Sci Rep. 2017;7:40509.

87. Ishimaru Y, Tomonari S, Matsuoka Y, Watanabe T, Miyawaki K, Bando T, Tomioka K, Ohuchi H, Noji S, Mito T. TGF- $\beta$ signaling in insects regulates metamorphosis via juvenile hormone biosynthesis. Proc Natl Acad Sci U S A. 2016;113(20):5634-9.

88. Kirilly D, Wong JJ, Lim EK, Wang Y, Zhang H, Wang C, Liao Q, Wang H, Liou YC, Yu F. Intrinsic epigenetic factors cooperate with the steroid hormone ecdysone to govern dendrite pruning in Drosophila. Neuron. 2011;72(1):86-100.

89. Roy A, George S, Palli SR. Multiple functions of CREB-binding protein during postembryonic development: identification of target genes. BMC Genomics. 2017;18(1):996.

90. Saha TT, Shin SW, Dou W, Roy S, Zhao B, Hou Y, Wang XL, Zou Z, Girke T, Raikhel AS. Hairy and Groucho mediate the action of juvenile hormone receptor Methoprene-tolerant in gene repression. Proc Natl Acad Sci U S A. 2016;113(6):E735-43

91. Henchcliffe C, García-Alonso L, Tang J, Goodman CS. Genetic analysis of laminin a reveals diverse functions during morphogenesis in Drosophila. Development. 1993;118(2):325-37.

92. Dolezelova E, Zurovec M, Dolezal T, Simek P, Bryant PJ. The emerging role of adenosine deaminases in insects. Insect Biochem Mol Biol. 2005;35(5): 381-9.

93. Van der Zee M, Da Fonseca RN, Roth S. TGF $\beta$ signaling in Tribolium: vertebrate-like components in a beetle. Dev Genes Evol. 2008;218(3-4):203-13.

94. Huang J, Tian L, Peng C, Abdou M, Wen D, Wang Y, Li S, Wang J. DPPmediated TGF $\beta$ signaling regulates juvenile hormone biosynthesis by activating the expression of juvenile hormone acid methyltransferase. Development. 2011;138(11):2283-91.
95. Zarin KN, Yang X, Bao R, Friedrich F, Beutel R, Friedrich M. The Pax gene eyegone facilitates repression of eye development in Tribolium. EvoDevo. 2011;2(1):8.

96. Tang B, Chen J, Yao Q, Pan Z, Xu W, Wang S, Zhang W. Characterization of a trehalose-6-phosphate synthase gene from Spodoptera exigua and its function identification through RNA interference. J Insect Physiol. 2010;56(7): 813-21

97. Chen J, Tang B, Chen H, Yao Q, Huang X, Chen J, Zhang D, Zhang W. Different functions of the insect soluble and membrane-bound trehalase genes in chitin biosynthesis revealed by RNA interference. PLoS One. 2010; 5(4):e10133.

98. Chen Q, Jin S, Zhang L, Shen Q, Wei P, Wei Z, Wang S, Tang B. Regulatory functions of trehalose-6-phosphate synthase in the chitin biosynthesis pathway in Tribolium castaneum (Coleoptera: Tenebrionidae) revealed by RNA interference. Bulletin of Entomol Res. 2017:1-12.

99. Lin $X$, Zhang $L$, Jiang $Y$. Distinct roles of met and interacting proteins on the expressions of takeout family genes in Brown Planthopper. Front Physiol. $2017 ; 8$.

100. Z-p K, Huang J, Tobe SS, X-I Y. A potential insect growth regulator: synthesis and bioactivity of an allatostatin mimic. Peptides. 2009;30(7):1249-53.

101. Martin D, Piulachs M, Bellés X. Inhibition of vitellogenin production by allatostatin in the German cockroach. Mol Cell Endocrinol. 1996;121(2):191-6.

102. Mayoral JG, Nouzova M, Navare A, Noriega FG. NADP+-dependent farnesol dehydrogenase, a corpora allata enzyme involved in juvenile hormone synthesis. Proc Natl Acad Sci U S A. 2009;106(50):21091-6.

103. Rewitz K, Yamanaka N, O'Connor MB. Ecdysone control of developmental transitions: lessons from Drosophila research. Annu Rev Entomol. 2013;58: 497-516.

104. Helvig C, Koener J, Unnithan G, Feyereisen R. CYP15A1, the cytochrome P450 that catalyzes epoxidation of methyl farnesoate to juvenile hormone III in cockroach corpora allata. Proc Natl Acad Sci U S A. 2004:101(12):4024-9.

105. Guittard E, Blais C, Maria A, Parvy J-P, Pasricha S, Lumb C, Lafont R, Daborn PJ, Dauphin-Villemant C. CYP18A1, a key enzyme of Drosophila steroid hormone inactivation, is essential for metamorphosis. Dev Biol. 2011;349(1):35-45.

106. Sutherland T, Unnithan G, Andersen J, Evans P, Murataliev M, Szabo L, Mash E, Bowers W, Feyereisen R. A cytochrome P450 terpenoid hydroxylase linked to the suppression of insect juvenile hormone synthesis. Proc Natl Acad Sci U S A. 1998:95(22):12884-9.

107. Darakananda K. The role of the hedgehog signaling pathway in the regulation of larval and adult appendage patterning in the flour beetle, Tribolium Castaneum. Honors Thesis Collection. 2014:205.

108. Süren-Castillo S, Abrisqueta M, Maestro JL. FoxO inhibits juvenile hormone biosynthesis and vitellogenin production in the German cockroach. Insect Biochem Mol Biol. 2012;42(7):491-8.

109. Bellés X, Martín D, Piulachs M-D. The mevalonate pathway and the synthesis of juvenile hormone in insects. Annu Rev Entomol. 2005;50:181-99.

110. Tanji T, Ip YT. Regulators of the toll and Imd pathways in the Drosophila innate immune response. Trends Immunol. 2005:26(4):193-8.

111. Noriega FG. Juvenile hormone biosynthesis in insects: what is new, what do we know, and what questions remain? Int Sch Res Notices. 2014:2014:16.

Ready to submit your research? Choose BMC and benefit from:

- fast, convenient online submission

- thorough peer review by experienced researchers in your field

- rapid publication on acceptance

- support for research data, including large and complex data types

- gold Open Access which fosters wider collaboration and increased citations

- maximum visibility for your research: over $100 \mathrm{M}$ website views per year

At $\mathrm{BMC}$, research is always in progress.

Learn more biomedcentral.com/submissions 